\title{
DESENVOLVIMENTO DE UM SISTEMA DE AQUISIÇÃO DE SINAIS ELETROMIOGRÁFICOS E UMA PRÓTESE 3D PARA O ENSINO DE ENGENHARIA BIOMÉDICA
}

Uriel A. Contardi - urielcontardi@ hotmail.com

Paulo R. Scalassara - prscalassara@utfpr.edu.br

Wagner Endo - wendo@utfpr.edu.br

Universidade Tecnológica Federal do Paraná

Avenida Alberto Carazzai, 1640

86300-000 - Cornélio Procópio - Paraná

Resumo: O presente trabalho visa o desenvolvimento de um módulo de ensino para aquisição de sinais eletromiográficos (EMG) aplicados em uma prótese $3 D$, executando uma integração dos sistemas e permitindo o controle e movimentação da prótese por meio dos sinais EMG. $O$ objetivo deste artigo é conceder uma plataforma didática para o suporte do ensino de Engenharia Biomédica na Engenharia Eletrônica em disciplinas que abordem a temática, aplicando o estudo teórico com uma contextualização prática.

Palavras-chave: Eletromiografia, Prótese, Sistema didático, Ensino de engenharia Biomédica.

\section{INTRODUÇÃO}

A Engenharia Biomédica é um campo no Brasil que está em constante crescimento. Porém, temos ainda poucos profissionais com entendimento aprofundado nessa área do saber. Outro problema enfrentado pelos alunos que acabam estudando essa área é a falta de aulas práticas. Dessa forma fica evidente a necessidade de novas metodologias para o ensino de Engenharia Biomédica que sejam capazes de proporcionar um melhor ensino e aproximação dos conteúdos teóricos com experiências práticas, preparando os profissionais para o cenário atual que envolve muitas mudanças tecnológicas e sociais. Assim, a área biomédica deve ser estudada de uma forma prática, por intermédio de uma perspectiva pedagógica que faça com que o aluno tenha uma vivência com o "fazer" (ANDRIGHETTO et al, 2007).

Existe ainda um descompasso entre a importância da área de Engenharia Biomédica e as estruturas curriculares em Engenharia Eletrônica no país. Ainda é escasso disciplinas que abordem o conteúdo de forma sistemática e prática nos cursos regulares de graduação e pósgraduação. Na Universidade Tecnológica Federal do Paraná (UTFPR), por exemplo, existem apenas duas disciplinas que tratam conteúdos nessa área: "Princípios de Engenharia Biomédica" e "Engenharia Biomédica" nenhuma das quais possui laboratório para devidas práticas. No âmbito da pós-graduação tem-se somente a matéria de "Instrumentação Biomédica" que também não possui aulas práticas.

O principal objetivo deste trabalho é preencher as lacunas existentes no ensino das disciplinas de Engenharia Biomédica com o desenvolvimento de um protótipo de membros 
superiores (especificamente mão e antebraço) de baixo custo que seja controlado por meio de sinais eletromiográficos, nos cursos de graduação e pós-graduação da UTFPR em Engenharia Eletrônica para que os alunos tenham uma aplicação prática dos conceitos abordados teoricamente.

Este sistema e protótipo permitirão por meio da prática a aplicação do que foi estudado nos livros sobre aquisição e processamento de sinais eletromiográficos e instrumentação biomédica. Em nível de hardware, espera-se proporcionar ao aluno a compreensão e utilização das estruturas internas dos circuitos presentes na aquisição e condicionamento destes sinais.

\section{ELETROMIOGRAFIA}

A eletromiografia é o estudo dos sinais mioelétricos, esses são sinais provenientes dos potenciais de ação que percorrem as fibras musculares, o fenômeno ocorre sempre que o indivíduo realiza uma contração muscular (NAJARIAN; SPLINTER, 2012).

O estudo desse sinal tem contribuído muito para a evolução da medicina e possui como principal finalidade aplicações clínicas, como: detecção de patologias, detecção de lesões em unidades motoras e acompanhamento da evolução em tratamentos fisioterapêuticos. Uma outra aplicação que merece destaque é a utilização do sinal EMG para controle de próteses, permitindo a reabilitação e restauração de funções motoras de pessoas com membros amputados (ANDRADE, 2007).

A coleta de sinais pode ser realizada por meio de metodologia invasiva ou não invasiva. A primeira utiliza de eletrodos que perfuram a pele e possui um sinal com menor nível de ruído e melhor distinção de sinais de cada músculo. A segunda utiliza de eletrodos superficiais que não causam desconforto, apesar disso o sinal proveniente desse sensor exibe maior ruído e não apresenta seletividade do músculo, uma vez que capta os sinais provenientes de um grupo muscular e não de um músculo específico (WEBSTER, 2009).

Para o desenvolvimento deste trabalho a metodologia não invasiva é mais conveniente, pois a utilização do produto final se pauta em ambiente acadêmico para demonstrações didáticas, dessa forma os eletrodos superficiais poderão ser utilizados sem problemas.

Entre as características do EMG de superfície (sEMG) pode-se citar: amplitude sinal de até $5 m \mathrm{~V}$, faixa de frequência entre 20 a $500 \mathrm{~Hz}$, sendo que a maior parte da energia do sinal está entre 50 e $150 \mathrm{~Hz}$ (BRONZINO, 2006).

\section{DESENVOLVIMENTO E VALIDAÇÃO}

Uma vez realizado o estudo das características desses sinais e ressaltado sua importância no campo da medicina o desenvolvimento do projeto é explanado nas próximas seções.

\subsection{Desenvolvimento do sistema de aquisição}

Como mencionado, este trabalho aborda a aquisição do EMG de superfície, o sinal possui a largura de banda compreendida entre 20 e $500 \mathrm{~Hz}$. Dessa forma, componentes que estejam fora dessa escala de frequência são dispensáveis e podem até mesmo diminuir a qualidade do sinal. Sendo assim, deve-se utilizar de sistemas que condicionem o sinal, eliminando as componentes indesejáveis, possíveis ruídos e interferências. A metodologia para o desenvolvimento da plataforma de condicionamento de sinais adotada neste trabalho é apresentada na Figura 1. 
Figura 1 - Diagrama em blocos do sistema de condicionamento de sinais sEMG.

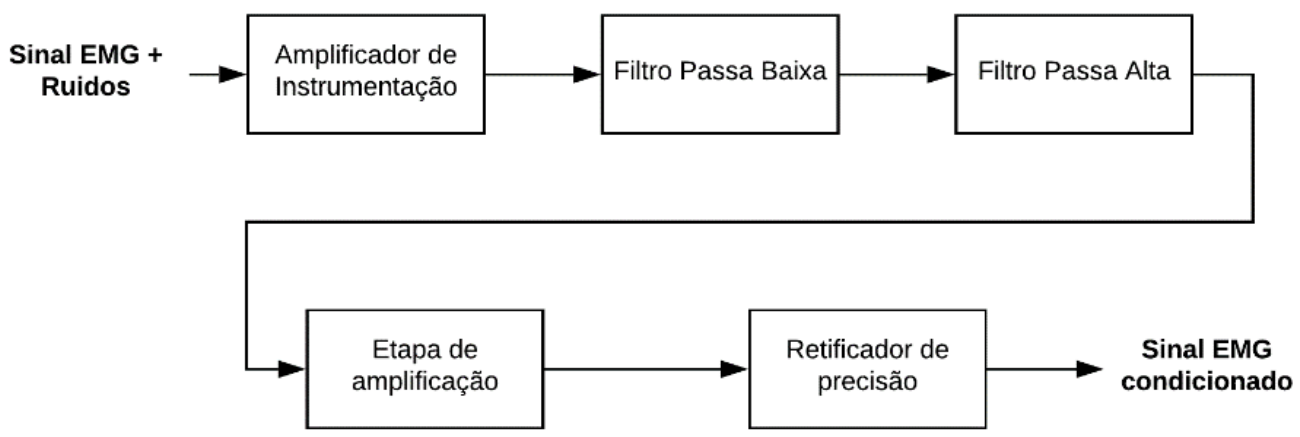

Fonte: Autoria própria (2020).

Como o sinal sEMG possui amplitudes pequenas o primeiro bloco é o de amplificação. Utilizou-se de um amplificador de instrumentação para esta tarefa, uma vez que esse possui características que facilitam sua utilização na instrumentação biomédica. Entre essas características pode-se citar: alta impedância de entrada, offset de saída mínimo, elevado ganho em malha aberta e elevada taxa de rejeição em modo comum (BECHELI, 2017).

O AD620, pertencente a Analog Devices, foi escolhido para este projeto, este CI permite o ajuste de ganho com apenas um resistor, facilitando a implementação do circuito. Além disso, visando facilitar ainda mais o ajuste do ganho, que pode variar de acordo com parâmetros externos, um trimmer foi utilizado no lugar da resistência. A Equação (1) apresenta o ganho do amplificador em função dessa resistência e a Figura 2 o diagrama esquemático do bloco de amplificação.

$$
G=\frac{49.4 K}{R_{g}}+1
$$

Figura 2 - Diagrama esquemático do bloco de amplificação.

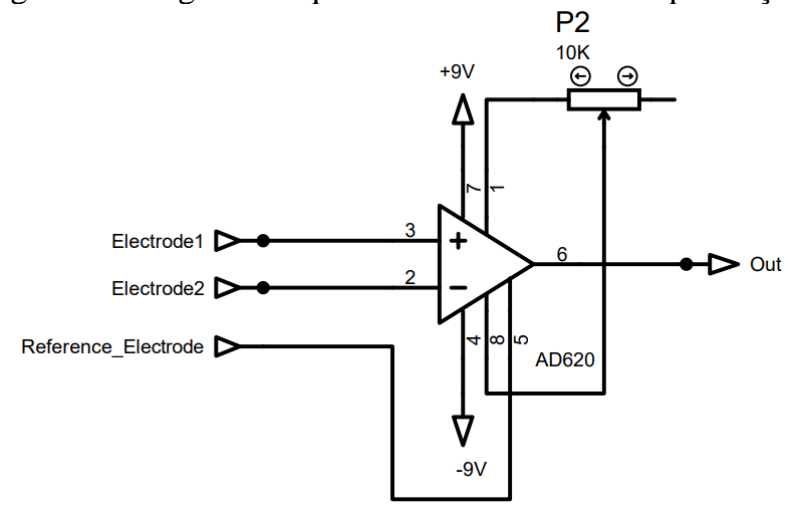

Fonte: Autoria própria (2020).

Para a etapa de filtragem do sinal escolheu-se filtros ativos devido a facilidade de projeto e possibilidade de amplificação do sinal de saída (SEDRA; SMITH, 2007).

A etapa do filtro passa baixa possui o objetivo de atenuar ruídos ou componentes de alta frequência indesejados. Optou-se pela utilização de um filtro de segunda ordem ativo com topologia de circuito Sallen-key (PERTENCE, 2015). 
O dimensionamento dos componentes foi realizado para garantir uma frequência de corte de $729 \mathrm{~Hz}$. O diagrama esquemático do filtro encontra-se na Figura 3.

Figura 3 - Diagrama esquemático do filtro passa baixa.

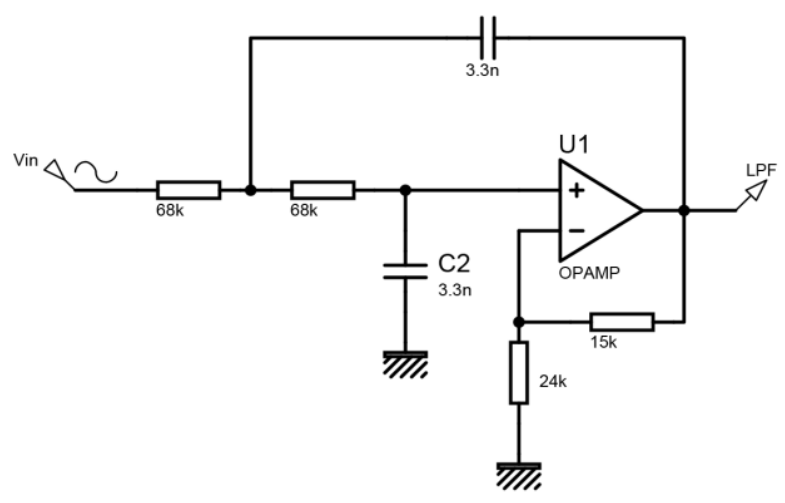

Fonte: Autoria própria (2020).

A Equação (2) apresenta a função de transferência do filtro em questão. Além disso, a resposta em frequência é mostrada na Figura 4.

$$
\frac{V_{\text {out }}(s)}{V_{\text {in }}(s)}=\frac{32,271 \times 10^{6}}{s^{2}+6,127 \times 10^{3} s+19,859 \times 10^{6}}
$$

Figura 4 - Resposta em frequência do filtro passa baixa.

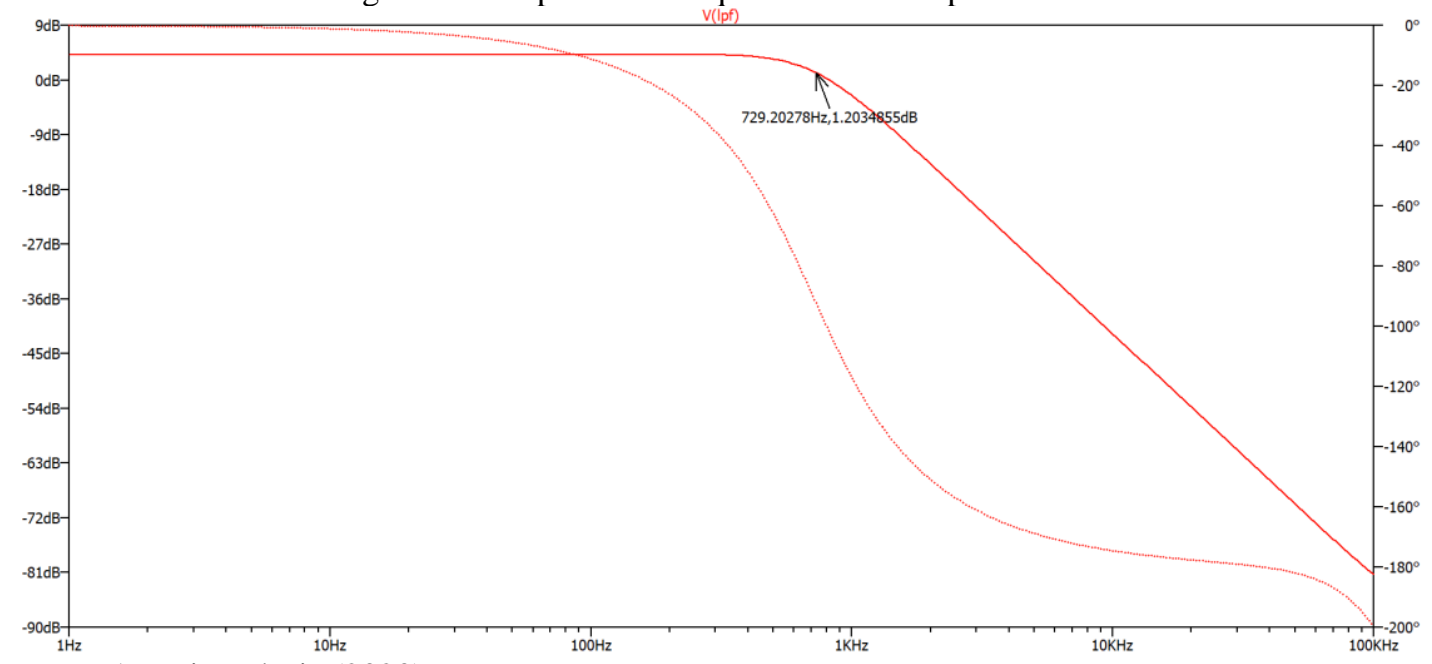

Fonte: Autoria própria (2020).

O filtro passa alta tem o propósito de atenuar/eliminar ruídos e interferência de baixa frequência, mas principalmente a componente DC offset, que é advinda da interação química entre Pele-Eletrodo. Utilizou-se também de um filtro de segunda ordem ativo com topologia de circuito Sallen-key.

O diagrama esquemático do filtro encontra-se na Figura 5, o dimensionamento dos componentes foi realizado para uma frequência de corte de $18 \mathrm{HZ}$. 
Figura 5 - Diagrama esquemático do filtro passa alta.

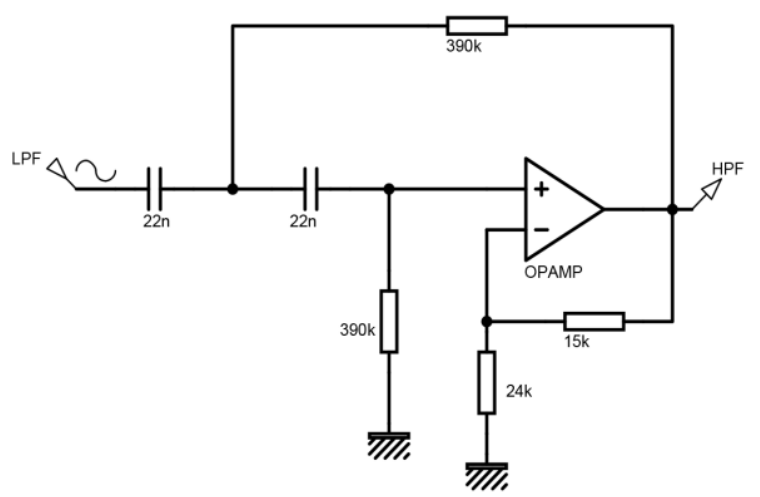

Fonte: Autoria própria (2020).

A Equação (3) apresenta a função de transferência do filtro. A resposta em frequência do filtro é mostrada na Figura 6.

$$
\frac{V_{\text {out }}(s)}{V_{\text {in }}(s)}=\frac{1,625 s^{2}}{s^{2}+160,256 s+13,583 \times 10^{3}}
$$

Figura 6 - Resposta em frequência do filtro passa alta.

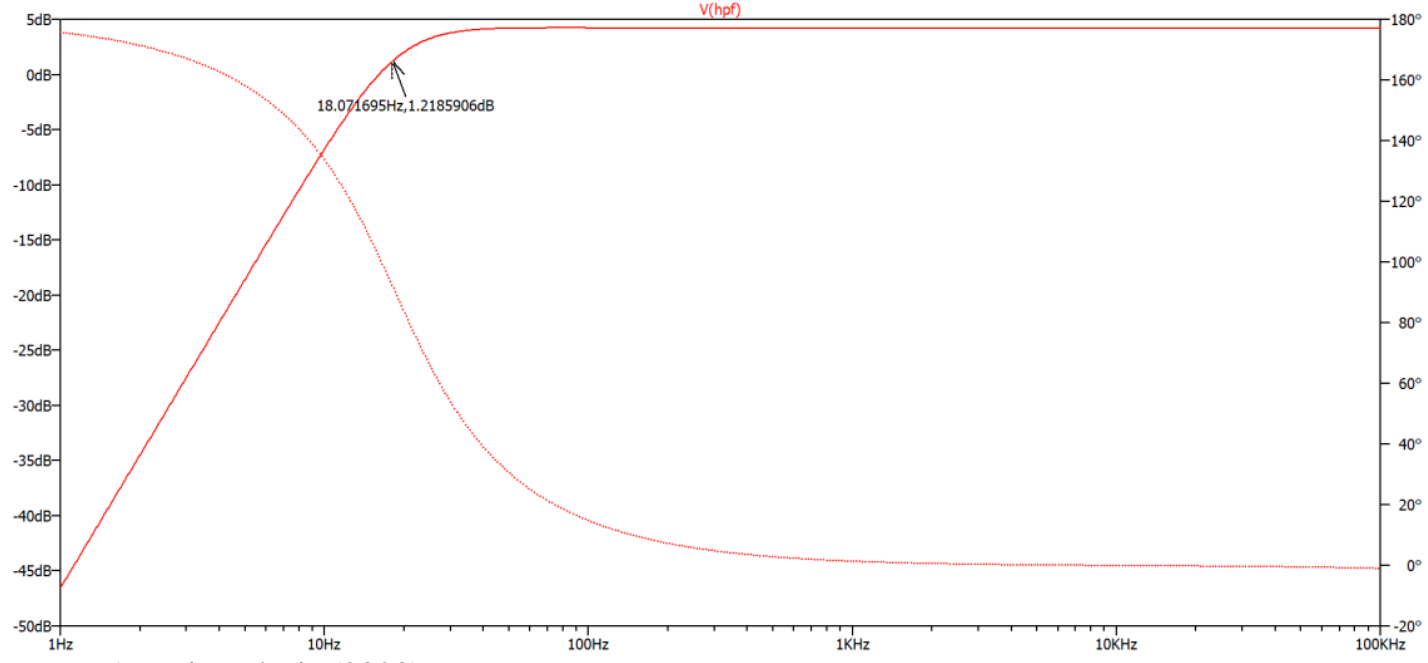

Fonte: Autoria própria (2020).

Após a filtragem, o sinal passa por mais um circuito de amplificação, para isso empregouse o uso de um amplificador com topologia inversora. Assim como na primeira etapa de amplificação um trimpot foi adicionado para controle do ganho. A Figura 7 apresenta o diagrama esquemático do amplificador. 
Figura 7 - Diagrama esquemático do amplificador inversor.

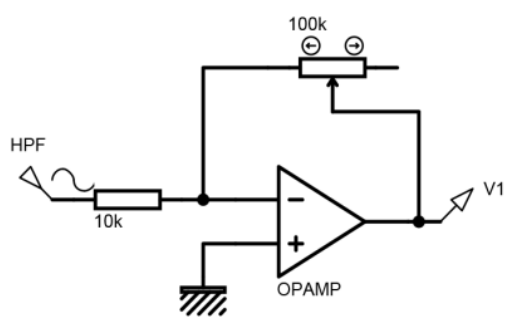

Fonte: Autoria própria (2020).

Os amplificadores operacionais possuem alimentação simétrica advinda de duas baterias de $9 \mathrm{~V}$ em série e conexão central como referência. Essas são utilizadas para isolar o sistema da rede elétrica e evitar ruídos provenientes desta. Todavia em aplicações com microcontroladores apenas a parcela positiva do sinal é utilizada, dessa forma um retificador de precisão foi empregado para eliminar a componente negativa do sinal EMG. A Figura 8 apresenta o circuito utilizado.

Figura 8 - Diagrama esquemático do retificador de precisão.

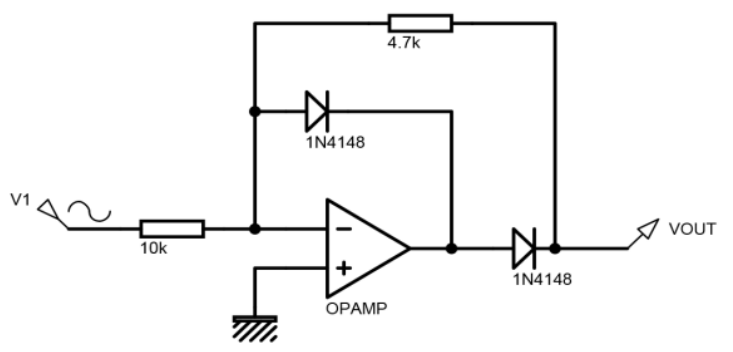

Fonte: Autoria própria (2020).

Com a finalidade de maior estabilidade e facilidade de utilização uma placa de circuito impressa (PCB) foi construída. A Figura 9 mostra o circuito da PCB. É possível notar que possui: máscara de solda na cor azul para evitar oxidações e alguns componentes SMD para reduzir o tamanho físico da placa. Um conector do tipo P2 foi escolhido para conexão do cabo dos eletrodos.

Figura 9 - Placa de circuito impressa do sistema de aquisição sEMG.

Fonte: Autoria própria (2020).

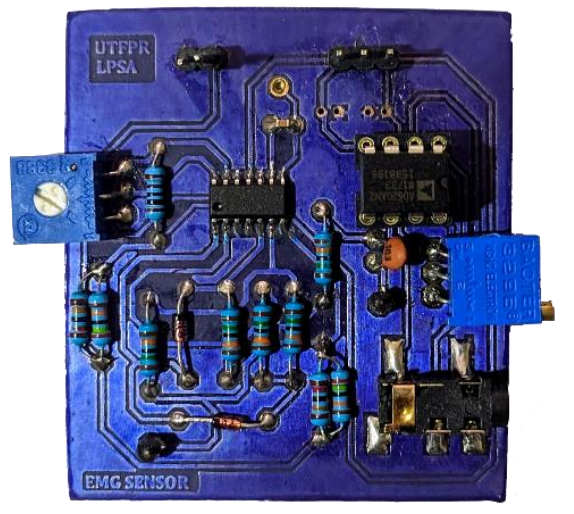




\subsection{Construção do protótipo da prótese}

Para proporcionar aos alunos uma maior experiência visou-se desenvolver uma prótese de membro superior, mais especificamente mão e antebraço. O protótipo possui por finalidade a visualização e se restringe a segurar pequenos objetos, sem possuir alto grau de liberdade e movimentação individual dos dedos.

Todo o projeto foi construído visando a redução de custo e facilidade de implementação. As peças foram impressas em impressora 3D, em material PLA.

O mecanismo de funcionamento utiliza de elásticos para flexibilidade e fio de nylon para movimentação. Este fio foi preso em cada dedo e é tracionado por um único servo motor. A Figura 10 apresenta o protótipo finalizado.

Figura 10 - Vista superior e inferior do protótipo.

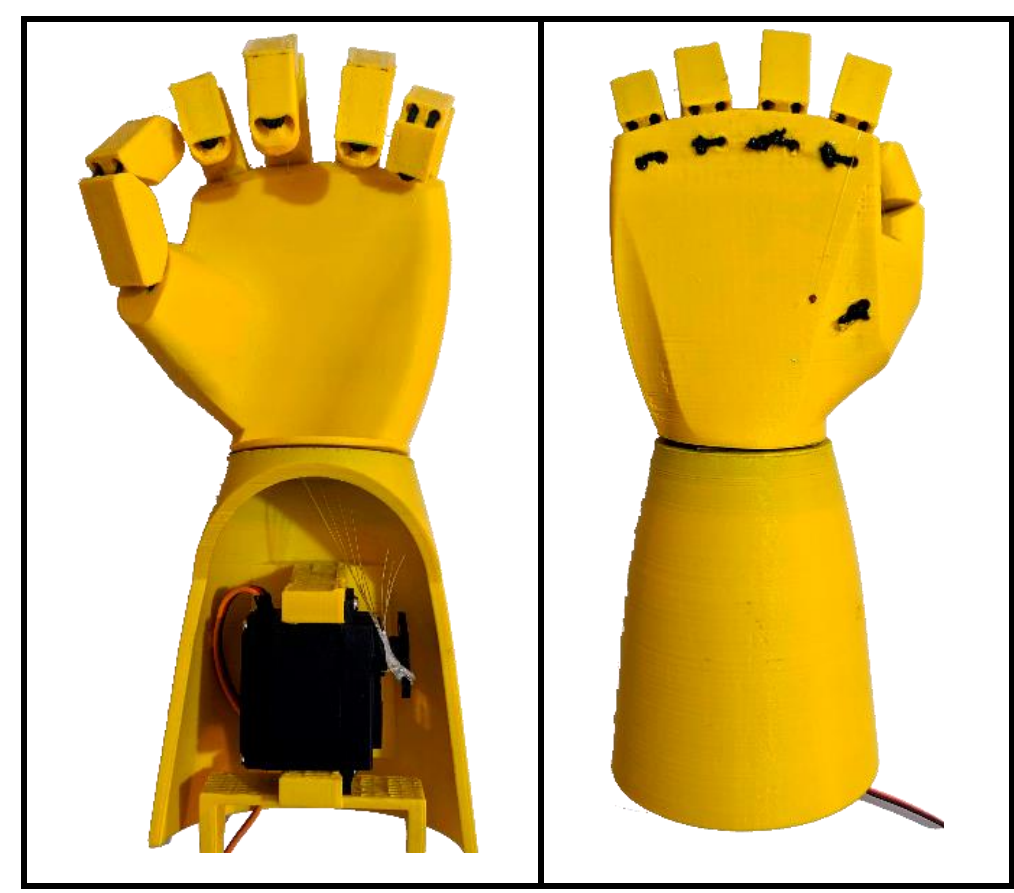

Fonte: Autoria própria (2020).

\subsection{Teste e validação}

Para validação da integração entre os sistemas a plataforma Arduino Uno foi utilizada, um firmware foi desenvolvido de acordo com o fluxograma da Figura 11.

Os eletrodos foram posicionados no antebraço. Vale notar que a amplitude do sinal aquisitado aumenta de acordo com a força exercida nos grupos musculares da região em que se encontra os sensores. Sendo assim, delimitou-se um valor de amplitude de tensão (threshold) que se ultrapassado deveria acionar o servo motor de forma a fechar a mão da prótese, caso o valor de amplitude fosse inferior a esse threshold o servo motor deveria voltar a posição inicial de forma a abrir a mão da prótese. 
Figura 11 - Fluxograma do firmware embarcado no Arduino Uno.

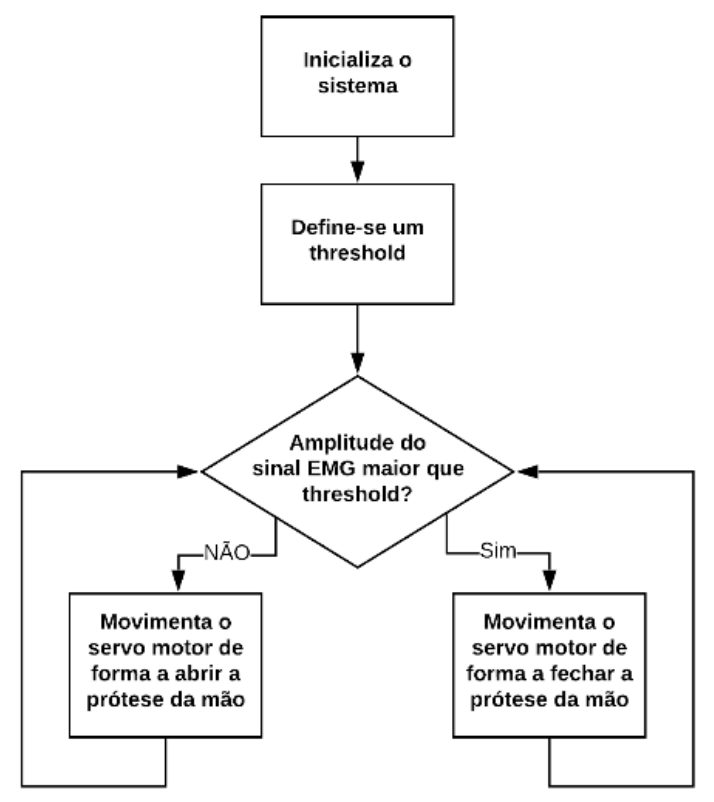

Fonte: Autoria própria (2020).

Após o ajuste dos ganhos das etapas de amplificação o sistema comportou-se como esperado e apresentou bons resultados.

\section{CONSIDERAÇÕES FINAIS}

O trabalho contemplou o desenvolvimento de um sistema de aquisição para sinais eletromiográficos e de um protótipo de prótese. Além disso realizou-se a integração de ambos os sistemas a fim de realizar o controle da prótese por meio de sinais sEMG.

O desenvolvimento do sistema de aquisição engloba conhecimentos das áreas de: instrumentação eletrônica, engenharia biomédica, processamento de sinais e projeto de circuitos eletrônicos.

Para o desenvolvimento do protótipo da prótese utilizou-se de conhecimentos da área de engenharia mecânica e engenharia de controle e automação, uma vez que envolveu a modelagem 3D de um objeto.

Observa-se que o projeto como um todo atinge diferentes áreas da engenharia, portanto pode-se afirmar que comtempla o âmbito da multidisciplinaridade.

Ressalta-se que o produto científico terá como finalidade utilização no ensino, seja por meio de pesquisas pertinentes ao trabalho, ou até mesmo na utilização em disciplinas que abordem a temática. Para essa última aplicação espera-se que o produto científico desenvolvido proporcione um melhor aprendizado com contextualização prática do ensino.

\section{Agradecimentos}

Agradecemos ao CNPq, Conselho Nacional de Desenvolvimento Científico e Tecnológico e aos pesquisadores do Laboratório de Processamento de Sinais e Aplicações (LPSA) da Universidade Tecnológica Federal do Paraná. 


\section{REFERÊNCIAS}

ANDRADE, N. A. Desenvolvimento de um sistema de aquisição e processamento de sinais eletromiográficos de superfície para utilização no controle de próteses motoras ativas. Universidade de Brasília, 2007.

ANDRIGHETTO, E. et al. Proposta de uma plataforma didática para o ensino de Engenharia Biomédica em Cursos de Graduação de Engenharia Elétrica: I Os sinais Bioelétricos. In: CONGRESSO LATINOAMERICANO DE INGENIERÍA BIOMÉDICA, 4., Porlamar. 2007.

BECHELI, Marcelo Haddad. Módulo de Eletromiografia. Orientador: Ruberlei Gaino. 2017. Dissertação (Bacharelado em Engenharia Elétrica) - Universidade Estadual de Londrina, [S. l.], 2017. Disponível em: http://www.uel.br/ctu/deel/TCC/TCC2017_MarceloHaddadBecheli.pdf. Acesso em: 29 jul. 2020.

BRONZINO, Joseph D. Biomedical Engineering Handbook: Medical Devices and Systems. 3. ed. Flórida: CRC Press, 2006.

NAJARIAN, Kayvan; SPLINTER, Robert. Biomedical Signal and Image Processing. 2. ed. atual. Flórida: CRC Press, 2012.

PERTENCE, A. Amplificadores Operacionais e Filtros Ativos. Editora Bookman, 2015.

SEDRA, Adel S.; SMITH , K. C. , Microeletrônica. 5a . Ed. São Paulo: Pearson Prentice Hall, 2007.

WEBSTER, John G. Medical Instrumentation. 4. Ed. John Wiley \& Sons, 2009.

\section{DEVELOPMENT OF AN ELECTROMYOGRAPHIC SIGNAL ACQUISITION SYSTEM AND A 3D PROSTHESIS FOR TEACHING BIOMEDICAL ENGINEERING IN GRADUATION AND POST- GRADUATION}

Abstract: The present work aims to the development of a teaching module for the acquisition of electromyographic signals (EMG) can be applied to a 3D prosthesis, using a system integration and allowing the prosthesis to be controlled by means of EMG signals. The purpose of this article is to provide a didactic platform to support the teaching of Biomedical Engineering in the electronic engineering course, applying the theoretical study in practice.

Keywords: Electromyography, Prosthesis, Didactic system, Biomedical engineering teaching. 